\title{
Gold(I)-catalyzed intermolecular hydroarylation of allenes with nucleophilic arenes: scope and limitations
}

\author{
Michael A. Tarselli, Ann Liu, and Michel R. Gagné* \\ Caudill Laboratories, University of North Carolina, Chapel Hill, NC 27599, USA
}

\begin{abstract}
The addition of nucleophilic methoxyarenes to allenes proceeds at room temperature in dichloromethane with a catalytic amount of phosphite-gold(I) precatalyst and silver additive. The addition is regioselective for the allene terminus, and generates $E$-allylation products without the need for prefunctionalization of the synthons as organometallics or allyl bromides. Coordinating heteroaromatics and sterically hindered allenes do not participate in the reaction.
\end{abstract}

\section{Introduction}

A major goal of the last decade in organic chemistry has been the development of $\mathrm{C}-\mathrm{C}$ bond-forming reactions that take place at room temperature without the prefunctionalization of either coupling partner.1 These reactions ideally proceed with catalytic quantities of readily available metals at room temperature. This research details the development of an allene addition to electronrich arenes catalyzed by gold(I), resulting in products normally accessed by deprotonation/allylation with an allylic halide.

Additions of pronucleophiles to allenes by gold catalysts have received much recent attention. Intramolecular cyclization of covalently linked allene nucleophiles by cationic gold(I) or gold(III) have been widely reported2,3 (Scheme 1), but the corresponding intermolecular variants are rare. Widenhoefer recently reported an intermolecular hydroalkoxylation of allenes, 4 and Yamamoto an intermolecular hydroamination.5 Intermolecular hydroarylations of alkynes were achieved by Reetz6 and He7 with gold(III) catalysts and Hashmi8 with gold(I) (Scheme 2), but, with the exception of recent results by Widenhoefer with methylated indoles, 9 there have not been any reports on gold-catalyzed intermolecular hydroarylation of allenes.

Our group recently reported an intramolecular hydroarylation of allenes utilizing a triphenylphosphite-derived gold(I) catalyst.10,11 One byproduct from a substrate intermediate was bis(allenyl)malonate, 12 which we discovered could react with electronrich 1,3,5-trimethoxybenzene 1 under the aforementioned conditions (Scheme 3) to form a hydroarylative product in trace amounts $(<10 \%)$.

Crude proton NMR and GC-MS analysis indicated that only one of the two allenes participated in the reaction, indicating that substrates possessing a single allene would participate. The commercially available 3-methyl-1,2-butadiene (dimethylallene, 2)was

(c) 2008 Elsevier Ltd. All rights reserved.

"Corresponding author. CB \#3290 Caudill Labs, Department of Chemistry, UNC, Chapel Hill, NC 27599, USA. mgagne@unc.edu (M.R. Gagné). . 
chosen as a commercially available screening substrate, and the results of reaction optimization are shown in Table 1.

Entries 1-5 indicate a preference for dichloromethane as solvent in this reaction, with more coordinating or nonpolar solvents markedly slowing reactivity. Interestingly, gold(I) precatalysts that are found to be optimal in other gold-catalyzed reactions (entries 613 and 714) were less active in this reaction. The trend toward more electrophilic phosphite ligands is evidenced when comparing entries 10,11, and 13. The optimal system of (4$\mathrm{ClPhO})_{3} \mathrm{P}(\mathrm{AuCl}) 15$ and $\mathrm{AgBF}_{4}$ reached full conversion after $1 \mathrm{~h}$, when employing allene as the limiting reagent in the presence of 2 mol equiv of arene. Control experiments between 1 and ethyl-2,3-butadienoate or dimethylallene ruled out silver and acid-mediated catalysis in this reaction.16 Another control indicated that gold(III)—either alone or with 3.0 equiv of silver cocatalyst—does not catalyze this reaction.

\section{Substrate scope}

The results indicate that small and relatively unsubstituted allenes work best in this chemistry. Functional groups such as esters, ethers, and enoates were well-tolerated, but more Lewis-basic heteroaromatic substrates did not function well (vide infra).

To probe how the electronics of the aryl system affected the ability to add an equivalent of allene, a variety of electron-rich methoxy-substitued arenes were tested. Shown in Table 2 are reactions utilizing allene $\mathbf{2}$, which generates products of traditional prenylation. Arenes possessing constructively oriented ortho- and para-directing groups gave higher yields. A preference for allylation at unhindered positions on the arene, presumably due to steric effects, is evidenced by the 6:1 ratio of products in $\mathbf{4}$ as well as the increased yield from $\mathbf{6}$ to 10.

The allene scope was next examined using $\mathbf{1}$ or $\mathbf{4}$ as the aryl nucleophile. Table 3 presents allenes that are found to participate in this chemistry. Allylic products were formed with $E$ stereochemistry, indicated by the ${ }^{1} \mathrm{H}$ coupling constants of the isolated products.

\section{Limitations}

While highly nucleophilic methoxyarenes readily participate in this chemistry, it was envisioned that the method would become more practical if it was tolerant of heterocyclic rings, such as indoles, furans, and pyrroles. Unfortunately, these reactions could not be advanced with the arenes shown in Figure 1 using heat or acid cocatalysts. Increasing the concentration of the reactants or even running the reaction in neat arene as solvent (methylpyrrole) did not result in useful conversion $(<10 \%)$.

Unlike previously reported methods by Hashmi, furans did not participate in either addition or phenol-rearrangement chemistry, instead decomposing to $>8$ products. One hypothesis for the failure of these reactions is that the cationic gold(I) catalyst is simply deactivated by the excess coordinating arene in the reaction. 17

Allenes with more sterically demanding $\alpha$ substituents were also unreactive in this chemistry. Scheme 4 lists allenes, which do not react under the examined conditions. Widenhoefer4 has proposed a mechanism, which could rationalize such an observation. This mechanism might also explain the low reactivity of substrate $\mathbf{1 7}$ relative to the parent allene 2; a $\sigma$-allyl gold species would be better stabilized by an internal $3^{\circ}$ carbocation (2) or a nearby heteroatomic group $(\mathbf{1 2}, \mathbf{1 5})$. Oshima and co-workers have noted a similar effect on allene substitution in the Mn-catalyzed allene allylation.18 


\section{Conclusion}

A method for the addition of methoxybenzenes to allenes by phosphite-gold(I) catalysts was reported. The reactions were stable to air and trace moisture, and were conducted on the benchtop in air. Electron-rich methoxybenzenes and unhindered mono-substituted allenes were found to be the best participants in these reactions.

\section{Experimental section}

\subsection{General}

All gold precatalysts were synthesized according to the published methods.4,13,14 Silver bistriflimide $\left(\mathrm{AgNTf}_{2}\right)$ was formed according to the procedure of Gagosz.19 All other silver salts were purchased from Strem Chemicals and stored in a nitrogen-atmosphere glovebox, then transferred to an oven-dried vial stored in a desiccator when used. All solvents were purified by alumina-packed columns under Ar or distillation from $\mathrm{Na}^{0}$ or $\mathrm{CaH}_{2}$. Arenes were purchased from Aldrich and used as received. Dimethylallene and ethyl-2,3butadienoate were purchased from Aldrich; allenylmalonate was synthesized according to the literature procedure. 20

\subsection{General procedure for intermolecular hydroarylation}

To a 5-mL vial charged with a stirbar were added (4-ClPhO) ${ }_{3-} \mathrm{PAuCl}(9.6 \mathrm{mg}, 15 \mu \mathrm{mol})$ and $\mathrm{AgBF}_{4}(3.0 \mathrm{mg}, 15 \mu \mathrm{mol})$, and dichloromethane $(1.0 \mathrm{~mL})$ by syringe, resulting in a light gray suspension. After stirring for $2 \mathrm{~min}, 1,3,5$-trimethoxybenzene $(100 \mathrm{mg}, 0.6 \mathrm{mmol})$ was added, resulting in a color change to light orange. After stirring for additional $2 \mathrm{~min}$, dimethylallene ( $20.0 \mathrm{mg}, 0.3 \mathrm{mmol}$ ) was added dropwise by microsyringe. Stirring was continued until GC/TLC (product $R_{f} 0.5$ in 1:7 ethyl acetate/hexanes) analysis indicated complete consumption of the allene. The reaction mixture was concentrated, loaded directly onto a silica flash column, and eluted with 1:10 to 1:8 ethyl acetate/hexanes to yield $\mathbf{3}$ (67\% yield) as a clear oil. ${ }^{1} \mathrm{H}$ NMR (300 MHz): $\delta 6.13(\mathrm{~s}, 2 \mathrm{H}), 5.16(\mathrm{t}, 1 \mathrm{H}), 3.79(\mathrm{~s}, 9 \mathrm{H}), 3.26(\mathrm{~d}$, $2 \mathrm{H}), 1.75$ (s, 3H), 1.65 (s, 3H). ${ }^{13} \mathrm{C}$ NMR (100 MHz): 159.9, 158.7, 130.6, 123.5, 111.0, $90.9,55.7,55.3,25.8,21.8,17.6$.

\subsection{Tri(4-chlorophenyl)phosphite gold(I) chloride}

White crystalline solid. ${ }^{1} \mathrm{H}$ NMR $\left(\mathrm{CDCl}_{3}, 300 \mathrm{MHz}\right): \delta 7.37(\mathrm{~d}, 2 \mathrm{H}, J=9 \mathrm{~Hz}), 7.12(\mathrm{dd}, 2 \mathrm{H}$, $\left.J_{1}=9 \mathrm{~Hz}, J_{2}=1.8 \mathrm{~Hz}\right) .{ }^{13} \mathrm{C}$ NMR (100 MHz): $\delta 147.6,132.5,130.6,122.3$ (d). ${ }^{31} \mathrm{P}(121$ $\mathrm{MHz}): \delta 112.0$.

\subsection{2-Methyl-4-(2,4-dimethoxyphenyl)-but-2-ene (5a) and 2-methyl-4-(2,6- dimethoxyphenyl)-but-2-ene (5b)}

6:1 Mixture, inseparable by column chromatography. Compound $5 \boldsymbol{a} .{ }^{1} \mathrm{H}$ NMR $(400 \mathrm{MHz})$ : $1.68(\mathrm{~s}, 3 \mathrm{H}), 1.71(\mathrm{~s}, 3 \mathrm{H}), 3.22(\mathrm{~d}, 2 \mathrm{H}), 3.77(\mathrm{~s}, 3 \mathrm{H}), 3.79(\mathrm{~s}, 3 \mathrm{H}), 5.26(\mathrm{t}, 1 \mathrm{H}), 6.42(\mathrm{~m}, 2 \mathrm{H})$, $7.01(\mathrm{~d}, 1 \mathrm{H})$. Compound $5 \boldsymbol{b} .{ }^{1} \mathrm{H}$ NMR $(400 \mathrm{MHz}): 1.65(\mathrm{~s}, 3 \mathrm{H}), 1.74(\mathrm{~s}, 3 \mathrm{H}), 3.32(\mathrm{~d}, 2 \mathrm{H})$, $3.79(\mathrm{~s}, 3 \mathrm{H}), 3.80(\mathrm{~s}, 3 \mathrm{H}), 5.19(\mathrm{t}, 1 \mathrm{H}), 6.53(\mathrm{~d}, 2 \mathrm{H}), 7.09(\mathrm{t}, 1 \mathrm{H}) .{ }^{13} \mathrm{C}$ NMR $(100 \mathrm{MHz})$ (mixture): 159.1, 158.1, 129.4, 123.3, 123.0, 122.6, 103.9, 98.6, 55.8, 55.3, 27.8, 25.8, 17.7.

\subsection{2-Methyl-4-(1,2,3,5-tetramethoxyphenyl)-but-2-ene (7)}

${ }^{1} \mathrm{H}$ NMR $(400 \mathrm{MHz}): \delta 6.24(\mathrm{~s}, 1 \mathrm{H}), 5.11(\mathrm{t}, 1 \mathrm{H}), 3.82(\mathrm{~s}, 3 \mathrm{H}), 3.81(\mathrm{~s}, 3 \mathrm{H}), 3.77(\mathrm{~s}, 3 \mathrm{H})$, $3.23(\mathrm{~d}, 2 \mathrm{H}), 1.72(\mathrm{~s}, 3 \mathrm{H}), 1.62(\mathrm{~s}, 3 \mathrm{H}) .{ }^{13} \mathrm{C}$ NMR $(100 \mathrm{MHz}): 153.6,152.4,151.6,130.7$, 123.6, 116.5, 100.0, 93.1, 60.9, 60.9, 56.3, 56.1, 25.7, 22.6, 17.7. HRMS (ESI $\left.{ }^{+}\right)$: expected 289.1416, observed $289.1422\left(\mathrm{M}+\mathrm{Na}^{+}\right)$. 


\subsection{2-Methyl-4-(1,2,3-trimethoxyphenyl)-but-2-ene (9)}

Clear oil. ${ }^{1} \mathrm{H}$ NMR $(400 \mathrm{MHz}): \delta 6.80(\mathrm{~d}, 1 \mathrm{H}), 6.59(\mathrm{~d}, 1 \mathrm{H}), 5.24(\mathrm{t}, 1 \mathrm{H}), 3.88(\mathrm{~s}, 3 \mathrm{H}), 3.86$ (s, 3H), 3.82 (s, 3H), 3.25 (d, 2H), 1.72 (s, 6H). ${ }^{13} \mathrm{C}$ NMR (100 MHz): 152.0, 151.8, 142.5, $131.9,127.9,123.5,123.3,107.5,60.7,56.0,28.2,25.7,17.1$.

\subsection{2-Methyl-4-(5-methyl-1,2,3-trimethoxyphenyl)-but-2-ene (11)}

${ }^{1} \mathrm{H}$ NMR (400 MHz): 1.66 (s, 3H), 1.75 (s, 3H), 2.22 (s, 3H), $3.26(\mathrm{~d}, 2 \mathrm{H}), 3.79-3.83(\mathrm{~m}$, 9H), $5.02(\mathrm{t}, 1 \mathrm{H}), 6.48(\mathrm{~s}, 1 \mathrm{H}) .{ }^{13} \mathrm{C}$ NMR $(100 \mathrm{MHz}): 151.8,151.1,140.5,131.8,131.0$, $126.3,123.2,109.6,60.9,60.8,55.9,25.6,19.6,17.8$.

\subsection{Dimethyl-2-(E-4-(1,3,5-trimethoxyphenyl)-but-2-enyl)malonate (13)}

Clear oil. ${ }^{1} \mathrm{H}$ NMR (300 MHz): $\delta 6.09(\mathrm{~s}, 2 \mathrm{H}), 5.58(\mathrm{dt}, 1 \mathrm{H}, J=15.3 \mathrm{~Hz}), 5.29(\mathrm{dt}, 1 \mathrm{H}$, $J=15.0 \mathrm{~Hz}), 3.78$ (s, 3H), 3.75 (s, 6H), 3.65 (s, 6H), 3.35 (t, $1 \mathrm{H}, J=7.5 \mathrm{~Hz}), 3.20$ (d, $2 \mathrm{H}, J=6$ $\mathrm{Hz}), 2.51(\mathrm{t}, 2 \mathrm{H}, J=14.7 \mathrm{~Hz}) .{ }^{13} \mathrm{C}$ NMR $(100 \mathrm{MHz}): 169.4,159.5,158.7,132.3,124.5$, 109.4, 90.8, 55.7, 55.3, 52.2, 52.1, 31.9, 25.6. HRMS (ESI ${ }^{+}$): expected 375.1482, observed $375.1482\left(\mathrm{M}+\mathrm{Na}^{+}\right)$.

\subsection{Dimethyl-2-(E-4-(1,3-dimethoxyphenyl)-but-2-enyl)-malonate (14a) and dimethyl-2-(E-4- (2,5-dimethoxyphenyl)-but-2-enyl)malonate (14b)}

6:1 mixture, inseparable by column chromatography. Compound 14a. ${ }^{1} \mathrm{H}$ NMR $(300 \mathrm{MHz})$ : $\delta 2.57(\mathrm{t}, 2 \mathrm{H}), 3.19(\mathrm{~d}, 2 \mathrm{H}), 3.40(\mathrm{t}, 1 \mathrm{H}), 3.67(\mathrm{~s}, 6 \mathrm{H}), 3.76(\mathrm{~s}, 6 \mathrm{H}), 5.40(\mathrm{dt}, 1 \mathrm{H}, J=15.2 \mathrm{~Hz})$, $5.63(\mathrm{dt}, 1 \mathrm{H}, J=15.2 \mathrm{~Hz}), 6.38(\mathrm{~m}, 2 \mathrm{H}), 6.94(\mathrm{~d}, 1 \mathrm{H}) .{ }^{13} \mathrm{C}$ NMR (100 MHz) (mixture): 169.4, $160.9,159.4,158.1,132.4,129.9,129.8,125.9,124.9,121.3,106.2,104.0,102.9,100.5$, 98.5, 55.7, 55.3, 55.3, 55.2, 52.2, 51.9, 32.2, 31.9, 15.2. HRMS (ESI): expected 345.1314, observed $345.1329\left(\mathrm{M}+\mathrm{Na}^{+}\right)$.

\subsection{Ethyl-E-4-(1,3,5-trimethoxyphenyl)-but-2-enoate (16)}

Clear oil. ${ }^{1} \mathrm{H}$ NMR (300 MHz): $\delta 7.01\left(\mathrm{dt}, 1 \mathrm{H}, J_{1}=15.6 \mathrm{~Hz}, J_{2}=6 \mathrm{~Hz}\right), 6.10(\mathrm{~s}, 2 \mathrm{H}), 5.66(\mathrm{dt}$, $1 \mathrm{H}, J=15.3 \mathrm{~Hz}), 4.11(\mathrm{~m}, 2 \mathrm{H}), 3.79(\mathrm{~s}, 3 \mathrm{H}), 3.76(\mathrm{~s}, 6 \mathrm{H}), 3.42(\mathrm{dd}, 2 \mathrm{H}), 1.23(\mathrm{t}, 3 \mathrm{H}) .{ }^{13} \mathrm{C}$ NMR (100 MHz): 167.2, 160.1, 158.8, 148.1, 129.3, 120.6, 116.7, 106.6, 104.2, 90.6, 59.9, 55.3, 25.5, 14.3. HRMS $\left(\mathrm{ESI}^{+}\right)$: expected 281.1389, observed $281.1387\left(\mathrm{M}+\mathrm{H}^{+}\right)$.

\subsection{1-(1,3,5-Trimethoxyphenyl)-2E-triskadecene (18)}

Clear oil. ${ }^{1} \mathrm{H}$ NMR (400 MHz): $\delta 0.85(\mathrm{t}, 4 \mathrm{H}), 1.22(\mathrm{~m}, 22 \mathrm{H}), 1.90(\mathrm{~m}, 2 \mathrm{H}), 3.22(\mathrm{~d}, 2 \mathrm{H})$, $3.79(\mathrm{~m}, 12 \mathrm{H}), 5.40(\mathrm{~m}, 2 \mathrm{H}), 6.12(\mathrm{~s}, 2 \mathrm{H}) .{ }^{13} \mathrm{C}$ NMR $(100 \mathrm{MHz}): 159.3,158.8,130.0,128.3$, 90.9, 55.8, 55.3, 32.5, 31.9, 29.6, 29.5, 29.3, 29.2, 25.7, 22.7, 14.1. HRMS $\left(\mathrm{ESI}^{+}\right)$: expected 371.2562, observed $371.2583\left(\mathrm{M}+\mathrm{Na}^{+}\right)$.

\section{Acknowledgments}

The authors gratefully acknowledge the National Institutes of Health Institute of General Medicine (GM-60578) for support of this research. We also thank John Gipson for the preparation of phenylallene and benzylallene (Scheme 4).

\section{References and notes}

1. Anastas, P.; Warner, J. Green Chemistry: Theory and Practice. Oxford University Press; New York, NY: 1998.

2. For recent (2007-2008) reviews concerning this topic, see: (a) Skouta R, Li C-J. Tetrahedron. 2008; 64:4917-4938. (b) Widenhoefer RA. Chem.-Eur. J. 2008; 14:5382-5391. (c) Gorin DJ, Toste FD. Nature. 2008; 446:395-403. [PubMed: 17377576] (d) Hashmi ASK. Chem. Rev. 2007; 107:31803211. [PubMed: 17580975] (e) Fürstner A, Davies PW. Angew. Chem., Int. Ed. 2007; 46:2-42. 
3. Watanabe T, Oishi S, Fujii N, Ohno H. Org. Lett. 2007; 9:4821-4824. [PubMed: 17924641]

4. Zhang Z, Widenhoefer RA. Org. Lett. 2008; 10:2079-2081. [PubMed: 18412355]

5. (a) Nishina N, Yamamoto Y. Angew. Chem., Int. Ed. 2006; 45:3314-3317. (b) Nishina N, Yamamoto Y. Synlett. 2007:1767-1770.

6. Reetz MT, Sommer K. Eur. J. Org. Chem. 2003:3485-3496.

7. Shi Z, He C. J. Org. Chem. 2004; 69:3669-3671. [PubMed: 15152995]

8. Hashmi ASK, Blanco MC. Eur. J. Org. Chem. 2006:4340-4342.

9. Toups KL, Liu GT, Widenhoefer RA. Unpublished results.

10. Tarselli MA, Gagné MR. J. Org. Chem. 2008; 73:2439-2441. [PubMed: 18278940]

11. For a recent phosphite-gold(I)-catalyzed hydroamination, see: Giner X, Najera C. Org. Lett. 2008; 10:2919-2922. [PubMed: 18553970]

12. Ogasawara M, Ikeda H, Nagano T, Hayashi T. Org. Lett. 2001; 3:2615-2617. [PubMed: 11483074]

13. Ref. 3, also Ref. 15.

14. López S, Herrero-Gómez E, Pérez-Galán P, Nieto-Oberhuber C, Echavarren AM. Angew. Chem., Int. Ed. 2006; 45:6029-6032.

15. Phosphites were synthesized by treatment of $\mathrm{PCl}_{3}$ with triethylamine ( 3.0 equiv) and a functionalized phenol (3.0 equiv) in THF overnight, followed by filtration over Celite. The corresponding 4-F and 4-Br analogs of the precatalyst were synthesized in this manner and found to be less active than the 4-Cl complex.

16. Catalysis with $2 \mathrm{~mol} \% \mathrm{TfOH} / \mathrm{CH}_{2} \mathrm{Cl}_{2}$ or $5 \mathrm{~mol} \% \mathrm{AgBF} 4$ in $\mathrm{CH}_{2} \mathrm{Cl}_{2}$ did not lead to any of the observed products. Addition of $5 \mathrm{~mol} \%$ (4-ClTPOP) $\mathrm{AuCl}$ to silver control after $24 \mathrm{~h}$ allows reaction to proceed normally, indicating an authentic gold-catalyzed reaction.

17. Herrero-Gómez E, Nieto-Oberhuber C, López S, Benet-Buchholz J, Echa-varren AM. Angew. Chem., Int. Ed. 2006; 45:5455-5459.

18. Nishikawa T, Shinokubo H, Oshima K. Org. Lett. 2003; 5:4623-4626. [PubMed: 14627399]

19. Mézailles N, Ricard L, Gagosz F. Org. Lett. 2005; 7:4133-4136. [PubMed: 16146370]

20. Zhang Z, Liu C, Kinder RE, Han X, Qian H, Widenhoefer RA. J. Am. Chem. Soc. 2006; 128:9066-9073. [PubMed: 16834380] 
<smiles>c1ccc2occc2c1</smiles><smiles>Cn1cccc1</smiles><smiles>COc1ccc(OC)cc1</smiles><smiles>COc1c(C(C)C)cccc1C(C)C</smiles><smiles>Cn1ccc2ccccc21</smiles><smiles>Cc1ccc(C)o1</smiles><smiles>COc1ccc(C(C)(C)C)cc1</smiles><smiles>Cc1cc(C)cc(C)c1</smiles><smiles>COc1cc(C)c(OC)c(C)c1C</smiles>

Figure 1.

Arenes tested, which are unreactive under the specified conditions. 


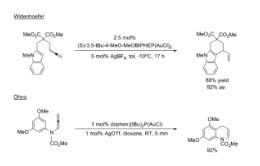

Scheme 1.

Tetrahedron. Author manuscript; available in PMC 2011 April 19. 


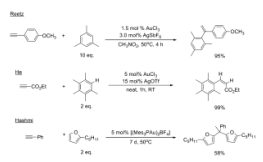

Scheme 2. 
Scheme 3 .

Tetrahedron. Author manuscript; available in PMC 2011 April 19. 

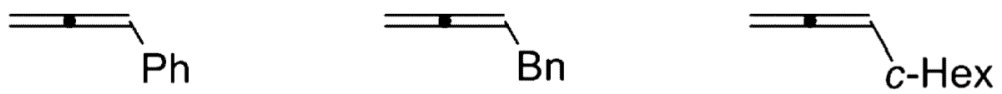

Scheme 4.

Allenes unreactive in the present system. 


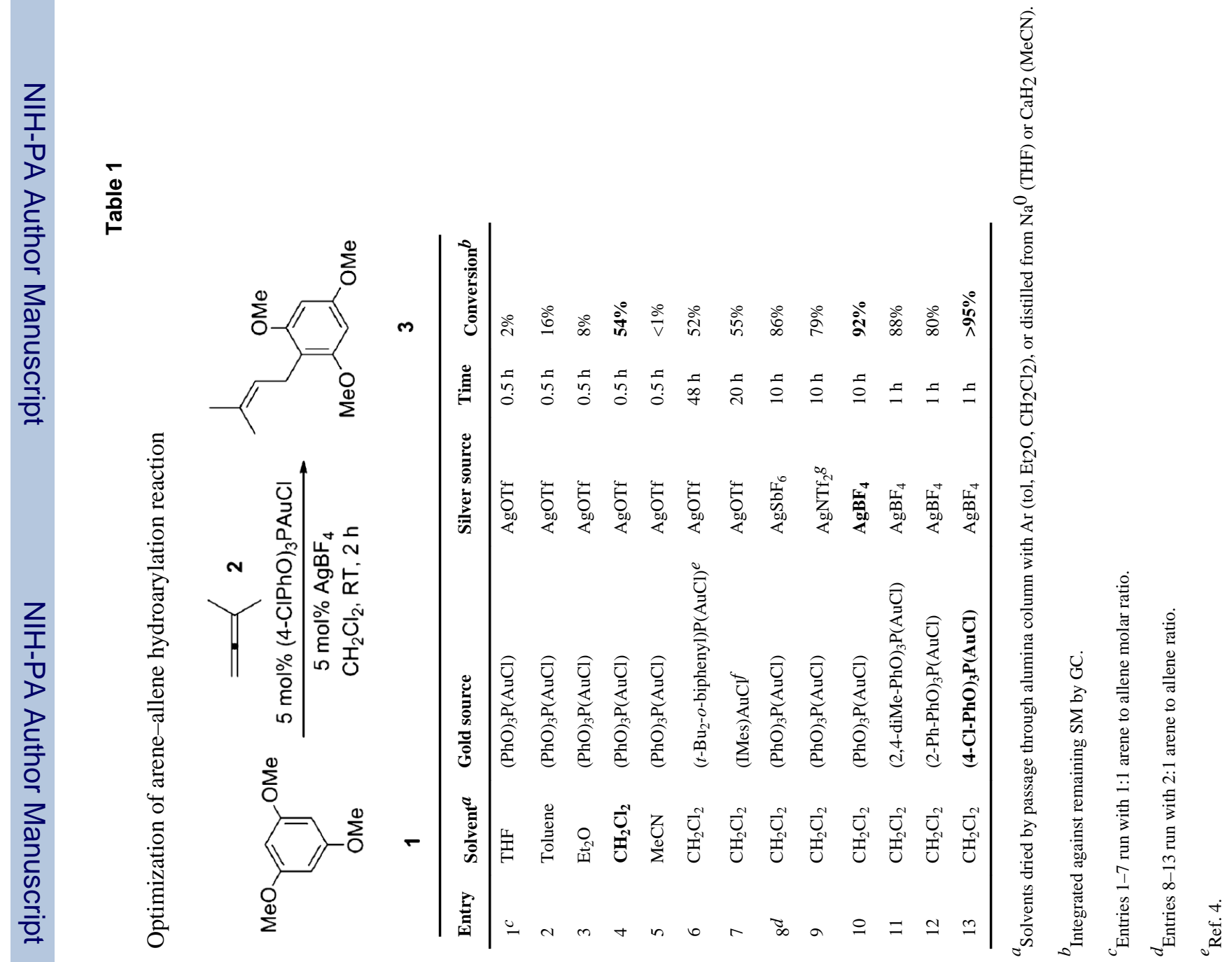


$\stackrel{n}{\rightarrow}$

感

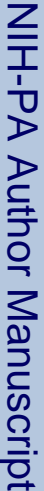


Table 2

Prenylation with dimethylallene

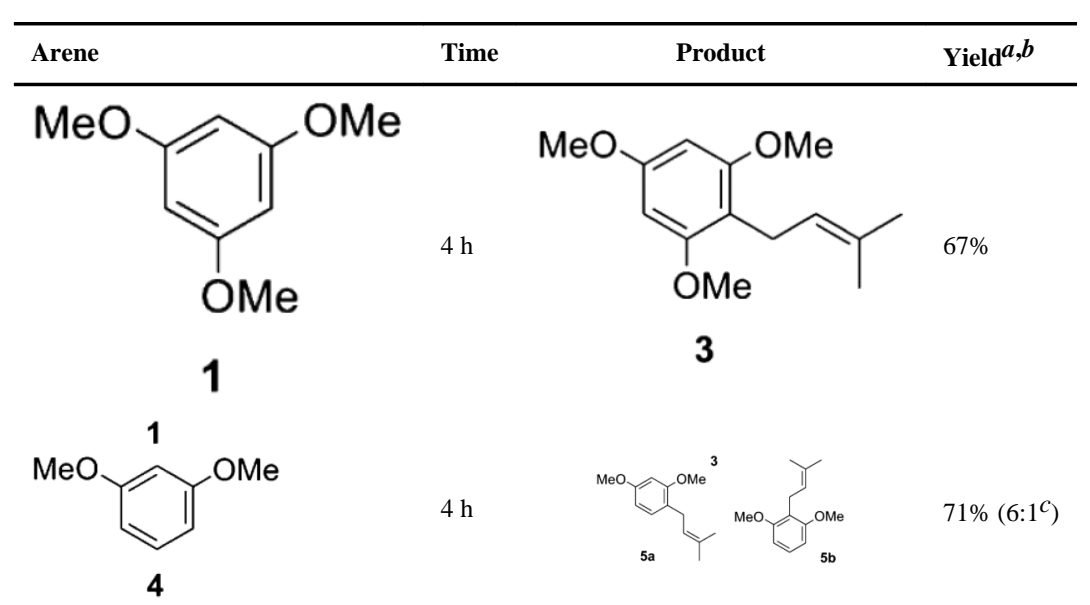<smiles>COc1cc(OC)c(OC)c(OC)c1</smiles><smiles>COc1cc(OC)c(OC)c(CC=C(C)C)c1OC</smiles><smiles>COc1cccc(OC)c1OC</smiles>

$16 \mathrm{~h}$<smiles>COc1ccc(CC=C(C)C)c(OC)c1OC</smiles>

$65 \%$

9

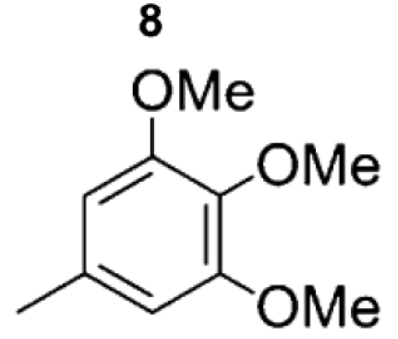

$12 \mathrm{~h}$<smiles>COc1cc(C)c(CC=C(C)C)c(OC)c1OC</smiles>
$75 \%$

11

10

\footnotetext{
${ }^{a}$ General procedure: 2.0 equiv arene and 1.0 equiv allene were added to $5 \mathrm{~mol} \%$ of preactivated gold catalyst in a 2-mL screwtop vial using anhydrous $\mathrm{CH}_{2} \mathrm{Cl}_{2}$ at room temperature for the time indicated.

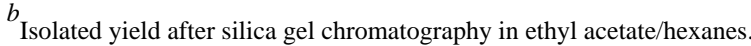

${ }^{c}$ Inseparable mixture.
} 
Table 3

Reaction of $\mathbf{1}$ and $\mathbf{4}$ with substituted allenes

Allene

12<smiles>C=CCCC(COC)C(OC)OC</smiles>

$14 \mathrm{~h}$
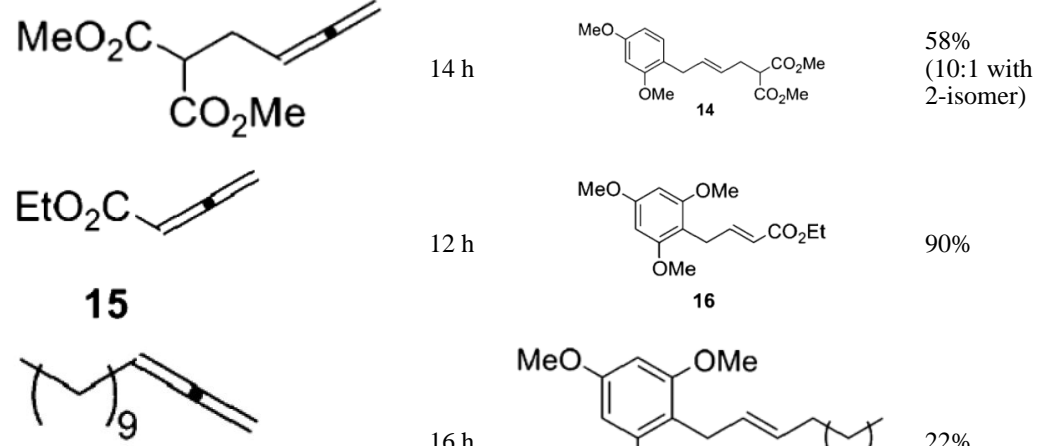

$12 \mathrm{~h}$

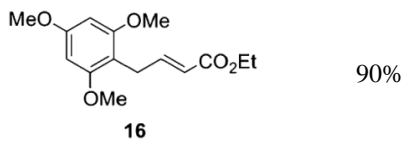

17

$16 \mathrm{~h}$

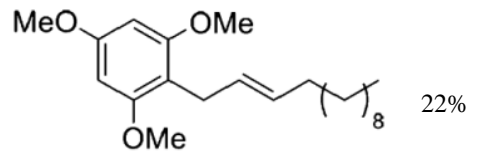

18 